\title{
Remote sensing of water cloud droplet size distributions using the backscatter glory: a case study
}

\author{
B. Mayer ${ }^{1}$, M. Schröder ${ }^{2}$, R. Preusker ${ }^{2}$, and L. Schüller ${ }^{3}$ \\ ${ }^{1}$ Deutsches Zentrum für Luft- and Raumfahrt (DLR), Oberpfaffenhofen, 82234 Wessling, Germany \\ ${ }^{2}$ Institut für Weltraumwissenschaften, Freie Universität Berlin, Carl-Heinrich-Becker Weg 6-10, 12165 Berlin, Germany \\ ${ }^{3}$ ESA, European Space \& Technology Centre (ESTEC), Keplerlaan 1, Postbus 299, 2200 AG Noordwijk, The Netherlands
}

Received: 4 March 2004 - Published in Atmos. Chem. Phys. Discuss.: 4 May 2004

Revised: 22 July 2004 - Accepted: 30 July 2004 - Published: 10 August 2004

\begin{abstract}
Cloud single scattering properties are mainly determined by the effective radius of the droplet size distribution. There are only few exceptions where the shape of the size distribution affects the optical properties, in particular the rainbow and the glory directions of the scattering phase function. Using observations by the Compact Airborne Spectrographic Imager (CASI) in $180^{\circ}$ backscatter geometry, we found that high angular resolution aircraft observations of the glory provide unique new information which is not available from traditional remote sensing techniques: Using only one single wavelength, $753 \mathrm{~nm}$, we were able to determine not only optical thickness and effective radius, but also the width of the size distribution at cloud top. Applying this novel technique to the ACE-2 CLOUDYCOLUMN experiment, we found that the size distributions were much narrower than usually assumed in radiation calculations which is in agreement with in-situ observations during this campaign. While the shape of the size distribution has only little relevance for the radiative properties of clouds, it is extremely important for understanding their formation and evolution.
\end{abstract}

\section{Introduction}

According to Houghton et al. (2001) probably the greatest uncertainty in future projections of climate arises from clouds and their interactions with radiation. A better understanding of clouds and the microphysical and optical properties is therefore crucial for improving the accuracy of climate predictions.

The optical properties of clouds are defined by the liquid water content and the droplet size distribution (and of course, the spatial distribution of these properties). The single scattering properties (extinction efficiency, single scat-

Correspondence to: Bernhard Mayer

(bernhard.mayer@dlr.de) tering albedo, and scattering phase function) for spherical water droplets are readily calculated with Mie theory, providing the size parameter $x=2 \pi r / \lambda$ and the complex refractive index of water as input ( $r$ is the droplet radius and $\lambda$ the wavelength). For a single droplet with given size, the scattering phase function exhibits considerable structure and resonances, characteristic for the particular size parameter. When averaged over a size distribution of an ensemble of droplets, these features are smoothed out. For typical cloud droplet size distributions, Hansen and Travis (1974) found in fact that the single scattering properties of water clouds are determined by the effective radius

$r_{\mathrm{eff}}=\frac{\int n(r) r^{3} d r}{\int n(r) r^{2} d r}$

of the size distribution $n(r)$ while the shape of the size distribution has only minor influence. The reason for this behaviour is that - at least in the limit of particles much larger than the wavelength - the effective radius is the extinctionweighted mean radius and hence a good measure for the extinction. Figure 1 illustrates this behaviour: Scattering phase functions have been calculated for different size distributions with an identical effective radius of $10 \mu \mathrm{m}$ using the Mie code of Wiscombe (1980). The corresponding phase functions nearly coincide everywhere, except for some angular regions: These are the backward and forward glories, around $180^{\circ}$ and $0^{\circ}$ scattering angle and the rainbow region, around $140^{\circ}$ (Laven, 2003). Obviously, some of the mentioned structures appearing in mono-disperse distributions are not completely smoothed out for narrow size distributions. These features might cause trouble in operational remote sensing methods which are generally based on the assumption of a constant size-distribution. While the shape of the size distribution obviously has only little impact on the single-scattering properties, it is important for the understanding of cloud formation, microphysics, and interaction with aerosol particles. Our results indicate that the size 

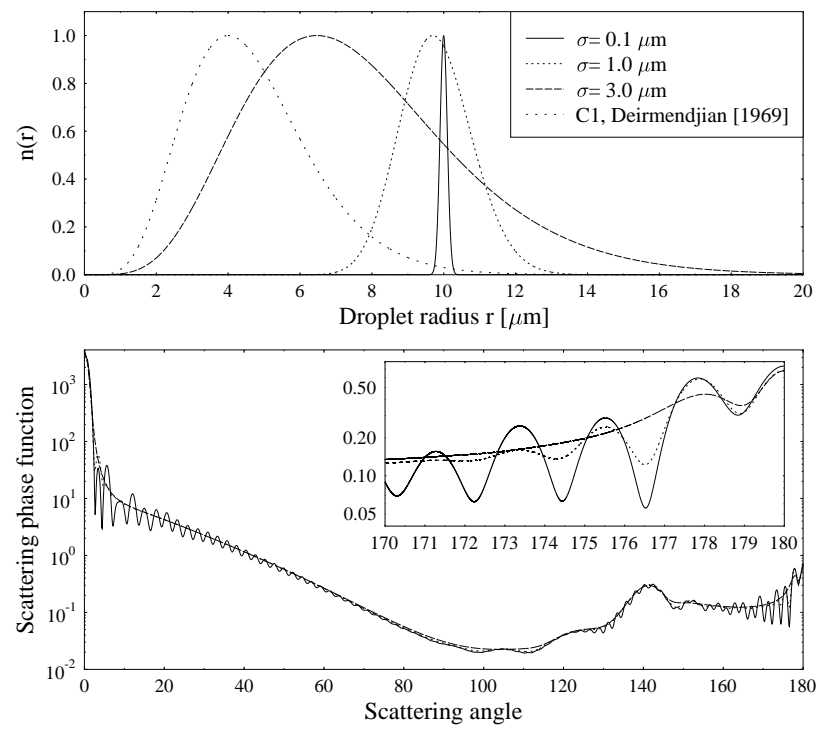

Fig. 1. (Top) Gamma droplet size distributions with different widths but identical effective radius $10 \mu \mathrm{m}$; the $\mathrm{C} 1$ size distribution by Deirmendjian (1969), with an effective radius of $6 \mu \mathrm{m}$, is also shown for comparison. (Bottom) Scattering phase functions for the three size distributions with effective radius $10 \mu \mathrm{m}$; the small graph shows a zoom into the $180^{\circ}$ backscatter direction.

distribution of stratocumulus clouds might actually be much narrower than generally assumed in radiation calculations.

In this study we use exactly those angular regions which might cause problems in usual remote sensing algorithms. Exploiting specific features of the single scattering properties in the $180^{\circ}$ backward direction, we gain insights into parameters which are not accessible by standard remote sensing techniques, in particular, the width of the size distribution. For this purpose, we exploit high-resolution observations by the Compact Airborne Spectrographic Imager (CASI) instrument operated onboard a Do-228 aircraft during the ACE-2 CLOUDYCOLUMN experiment (Brenguier et al., 2000a). Section 2 briefly describes the CASI instrument and the libRadtran model used in this study and outlines the method. In Sect. 3, the technique is illustrated in detail by evaluating a specific observation. In Sect. 4 the results are summarized and discussed.

\section{Data and forward simulations}

\subsection{Observations}

The Compact Airborne Spectrographic Imager (CASI) (Babey and Anger, 1989; Anger et al., 1994) is a "pushbroom" imaging spectrometer with a $34^{\circ}$ field of view across track. The spectral range from $430 \mathrm{~nm}$ to $970 \mathrm{~nm}$ can be covered with 512 pixels in the spatial axis and 288 spectral channels. During the ACE-2 campaign, CASI was op-

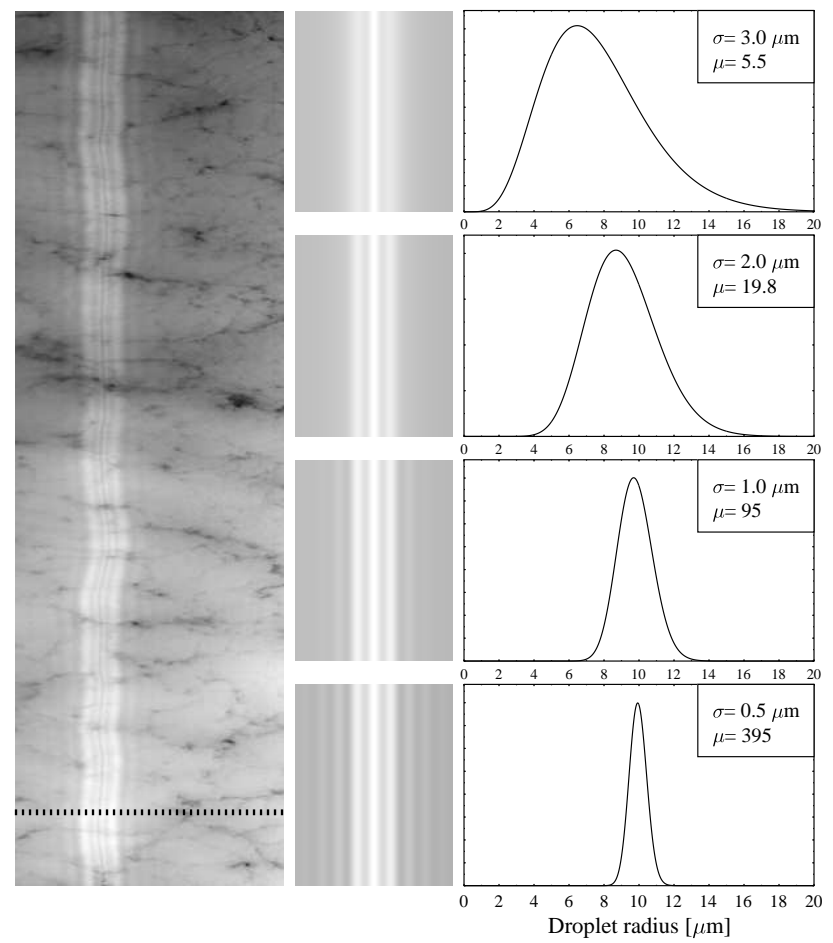

Fig. 2. CASI observation for 26 June 1997 (left); the length of the flight leg is $10 \mathrm{~km}$ and the width of the strip is $1.2 \mathrm{~km}$ at cloud top; the dark band in the center is the aicraft shadow. Simulated reflectivity distribution on the same angular grid (middle) for four different droplet size distributions (right) with identical effective radius $10 \mu \mathrm{m}$; the simulations do not include the aircraft shadow. The dotted line marks scan line 612 which will be studied in more detail later.

erated onboard the Do-228 aircraft to measure reflected solar radiation. The programmable channels were chosen to allow derivation of cloud albedo and optical thickness (with maximum spatial resolution) as well as cloud top height, using measurements within the Oxygen A band over 39 directions. For our study we used the $753 \mathrm{~nm}$ channel, with the full angular resolution of 512 pixels, each with $0.07^{\circ}$ width which translates to a spatial resolution of $2.5 \mathrm{~m}$ at cloud top across track. Along track, the resolution is determined by the speed of the aircraft and the sampling time, which evaluates to about $15 \mathrm{~m}$ per image pixel. For the quantitative analysis, a moving average over five scan lines along track was applied to the data which effectively reduces the resolution to $75 \mathrm{~m}$ along track, keeping the full detail of $2.5 \mathrm{~m}$ across track. The uncertainty of the radiance observation is estimated to be about $3 \%$.

The left panel of Fig. 2 shows an example of a CASI observation where the backscatter glory is clearly visible. The measurement was taken on 26 June 1997 for an airmass of marine origin (Brenguier et al., 2000a). The flight direction is from top to bottom of the image. The stripes are actually the backward glory, a phenomenon which is well known 
to frequent air-travellers: From an airplane, the glory is often visible as a bright ring around the aircraft shadow on a cloud. For the pushbroom scanning geometry of the CASI instrument, a cross section through the glory is only visible if (a) the sun is high in the sky (solar zenith angle smaller than $15^{\circ}$ because otherwise this feature is outside the scanning range of the nadir-looking instrument) and if (b) the flight direction is perpendicular to the solar principal plane (which it is in this case). That the instrument is looking exactly in the $180^{\circ}$ backscattering direction is proven by the dark line in the bright center stripe: This is the shadow of the aircraft.

\subsection{Simulations}

To simulate the CASI observations, multiple scattering calculations were done, using detailed cloud optical properties as input. To provide the cloud optical properties, extensive Mie calculations were required for various size distributions. For these calculations we used the well-tested code by Wiscombe (1980). A gamma distribution was assumed as size distribution which is a common assumption in cloud physics:

$n(r)=N_{0} \cdot r^{\mu} \cdot e^{-\mu \frac{r}{a_{0}}}$,

where $a_{0}$ is the mode radius, $\mu$ defines the shape of the distribution,

$N_{0}=\frac{\mu^{\mu+1}}{\Gamma(\mu+1) a_{0}^{\mu+1}}$

is a normalization constant, and $\Gamma(x)$ is the gamma function. Probably the most common assumption for a cloud droplet size distribution is the $\mathrm{C} 1$ size distribution by Deirmendjian (1969) which is a gamma distribution with $a_{0}=4 \mu \mathrm{m}$ and $\mu=6$ (see Fig. 1).

The effective radius of the gamma distribution evaluates to

$r_{\mathrm{eff}}=\frac{\int n(r) r^{3} d r}{\int n(r) r^{2} d r}=\frac{a_{0}}{\mu} \frac{\Gamma(\mu+4)}{\Gamma(\mu+3)}$

and the width $\sigma$ of the size distribution (which we simply define as the standard deviation) is

$\sigma=\sqrt{\int n(r)(r-<r>)^{2} d r}=\frac{a_{0}}{\mu} \sqrt{\mu+1}$.

For the $\mathrm{C} 1$ by Deirmendjian (1969), $\quad r_{\mathrm{eff}}=6 \mu \mathrm{m}$ and $\sigma=1.76 \mu \mathrm{m}$.

Various size distributions were sampled, with effective radii $r_{\text {eff }}$ between $4 \mu \mathrm{m}$ and $15 \mu \mathrm{m}$ and widths $\sigma$ between $0.1 \mu \mathrm{m}$ and $9 \mu \mathrm{m}$. For the calculation of the optical properties by integrating over the size distribution, Mie calculations were carried out with increments of $0.001 \mu \mathrm{m}$ in the radius range 0.001 to $30 \mu \mathrm{m}$. This sampling has been shown to be fine enough to correctly produce the scattering phase functions for the given size distribution: Further reduction of the step width did not change the phase functions anymore.
The scattering phase functions derived from these calculations were used as input to the radiative transfer model libRadtran by Kylling and Mayer (1993-2004). libRadtran is a flexible and user-friendly model package to calculate radiance, irradiance, and actinic flux for arbitrary input conditions. The model handles absorption and scattering of molecules and aerosols, as well as water and ice clouds. libRadtran provides a choice of radiative transfer solvers, including the discrete ordinate code DISORT by Stamnes et al. (1988) which we used for this study. In particular, version 2.0 of DISORT was used because it is more accurate in handling highly detailed phase functions. To make sure that all details are covered in the necessary angular resolution, the number of streams was set to 256. This high number of streams is essential, since radiances for a narrow angle range are to be calculated which, as our comparisons with less streams show, would be badly sampled and uncertain otherwise.

The influence of the atmosphere at $753 \mathrm{~nm}$ is very small because this wavelength is outside the important absorption bands. A calculation by libRadtran gives a vertically integrated optical thickness due to molecular absorption of only 0.004 for the Midlatitude summer atmosphere of Anderson et al. (1986), due to some absorption by oxygen, ozone, nitrogen dioxide, and water vapour. The vertically integrated optical thickness of molecular (Rayleigh) scattering is 0.027 . The albedo of the underlying water surface is about 0.02 , according to the parameterization of sea surface BRDF by Nakajima and Tanaka (1983) and Cox and Munk (1954), as implemented in libRadtran. Hence, this wavelength is ideally suited for the remote sensing of clouds. For the following study, only an isolated cloud layer is considered, neglecting surface albedo and background atmosphere. A test calculation for typical conditions revealed a difference between simulations including and excluding the background atmosphere and surface of less than $0.8 \%$ for the upward radiance at the location of the airplane.

The solar zenith angle for the observation to be studied was $10^{\circ}$, and the scan direction was the solar principal plane. Therefore, all following calculations are done for these conditions. Consequently, the $180^{\circ}$ backscatter direction (the center of the glory) corresponds to $-10^{\circ}$ in all plots.

The middle panel of Fig. 2 shows an example of the libRadtran simulation of the backward glory for four different size distributions. As can be expected from Fig. 1, the glory becomes more prominent for narrow size distributions. As the grey scales for observation and simulation were chosen identically, a mere visual inspection of the images reveals that the width of the size distribution needs to be in the order of $1.0 \mu \mathrm{m}$ or smaller in order to produce the three center maxima plus three side maxima which are visible in the data. The large $\mu$ 's of the gamma-distribution ( $\mu=95$ for a width of $1 \mu \mathrm{m}$ ) already indicate that this is much narrower than the widely-used standard C1 cloud model of Deirmendjian (1969) for which $\mu=6$. 

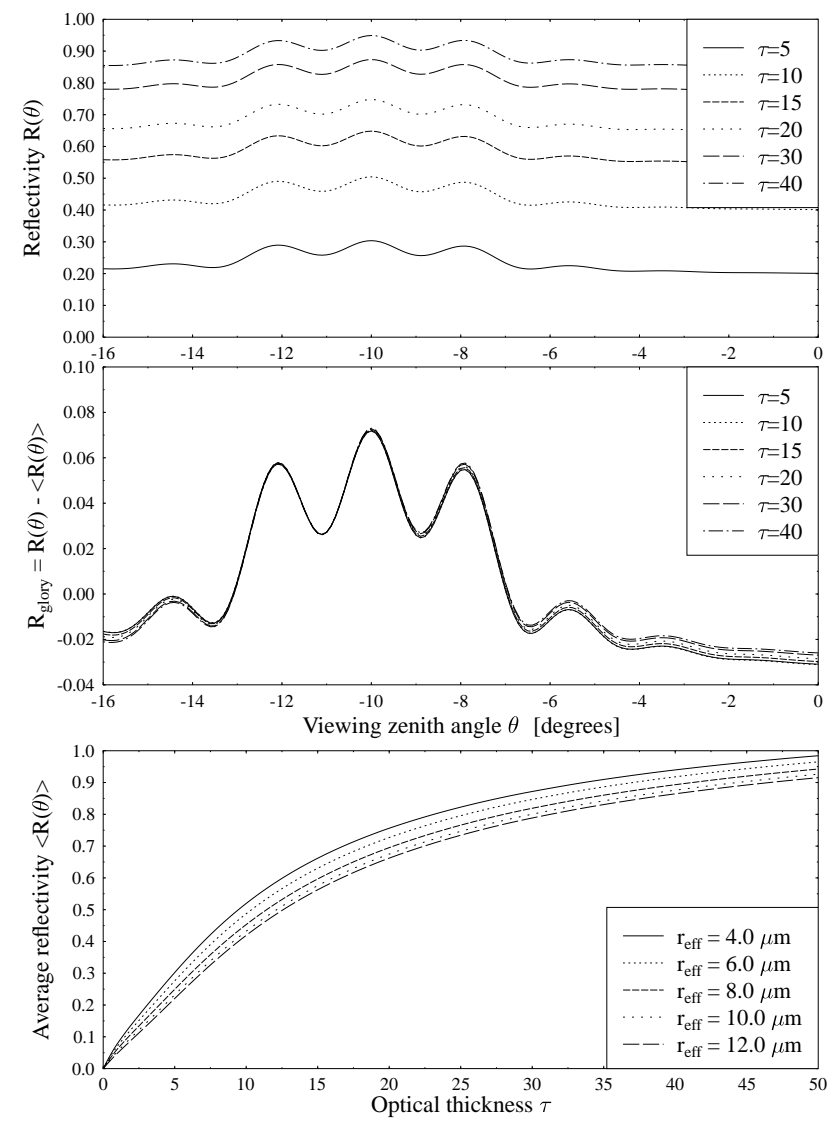

Fig. 3. (Top) Simulated reflectivity, for effective radius $10 \mu \mathrm{m}$ and width $1 \mu \mathrm{m}$. (Middle) Glory reflectivity, after subtraction of the average, see Eq. (6). (Bottom) Average reflectivity in the $180^{\circ}$ backscatter direction, as function of the optical thickness.

\section{Retrieval}

Our simulations of the reflectivity in the backscatter direction suggested that the shape of the glory is nearly independent of the optical thickness of the cloud, if the latter is larger than about 3, see top panel of Fig. 3. This led us to the definition of the "glory reflectivity" $R_{\text {glory }}(\theta)$ which isolates the shape of the glory by subtracting the average reflectivity $\langle R(\theta)>$ :

$R_{\text {glory }}(\theta)=R(\theta)-<R(\theta)>$,

where the average reflectivity $<R(\theta)>$ is simply

$<R(\theta)>=\frac{1}{N} \sum_{i=1}^{N} R\left(\theta_{i}\right)$

and $N$ is the number of data points. The middle panel of Fig. 3 clearly shows that the glory reflectivity $R_{\text {glory }}(\theta)$ curves practically coincide. The reason for this behaviour is easily understood: The glory is a single scattering feature, and if the cloud is optically thick enough to ensure that every photon entering the cloud is scattered at least once, the glory

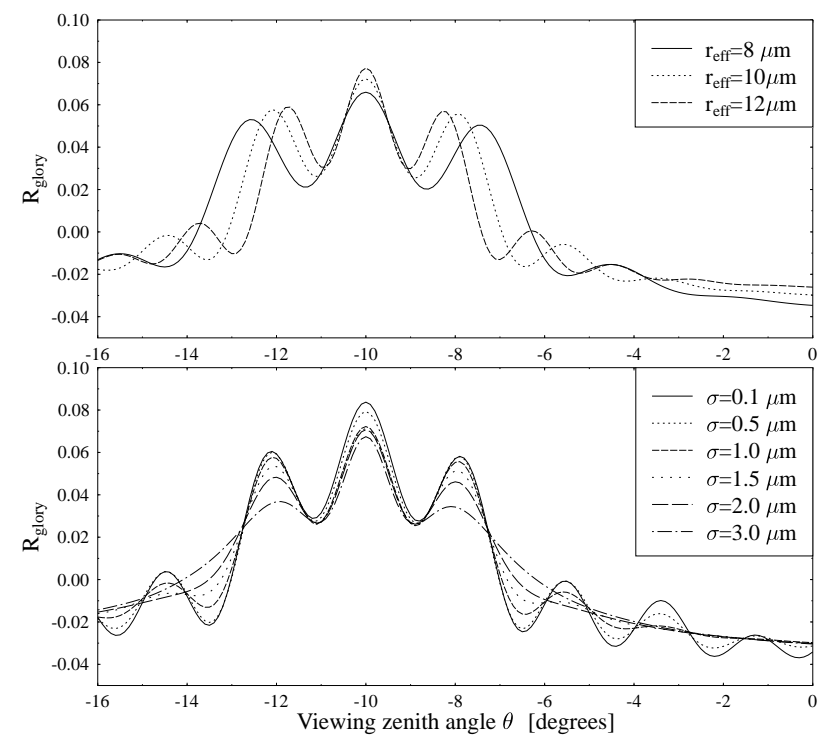

Fig. 4. (Top) The glory reflectivity for three different effective radii but constant width $1 \mu \mathrm{m}$. (Bottom) The glory as a function of the width of the size distribution for identical effective radius $10 \mu \mathrm{m}$.

structure should not change anymore. In our case, this is always given since for a cloud of optical thickness 3 , a fraction of $1-e^{-3 / \cos \left(\theta_{0}\right)}>0.95$ of the photons has undergone at least one scattering ( $\theta_{0}$ is the solar zenith angle). The glory structure sits on top of a multiple-scattering background which of course depends on optical thickness: The bottom plot of Fig. 3 shows the average reflectivity $\langle R(\theta)\rangle$, as a function of cloud optical thickness and for various effective radii. The curves show the usual behaviour of the cloud reflectivity outside the glory region: A strong dependence on optical thickness and only little sensitivity to the effective radius. As expected, the dependence on the width of the size distribution is even smaller and not plotted. In summary, the glory reflectivity $R_{\text {glory }}(\theta)$ is therefore a direct measure of the droplet size distribution while the average reflectivity contains the information about the optical thickness.

The top panel of Fig. 4 shows the glory reflectivity for three different effective radii. The distance of the maxima increases with decreasing droplet size. The bottom panel of Fig. 4 shows the glory reflectivity $R_{\text {glory }}(\theta)$ for an effective radius of $10 \mu \mathrm{m}$ and different widths of the size distribution. Two features are obvious: First, the maxima are more distinguished with decreasing width of the size distribution, as already mentioned. In particular, the side maxima next to the three main maxima are only visible for widths smaller than about $1-1.5 \mu \mathrm{m}$. Second, although the shape depends on the width of the size distribution, the distance between neighbouring maxima is almost constant for all investigated cases.

Let us summarize the four most important features of the glory, derived from radiative transfer calculations: First, the average reflectivity in the glory region depends mainly on 
optical thickness and only little on the droplet size. Second, the shape of the glory does not depend on the optical thickness of the cloud which lead us to the definition of the glory reflectivity, $R_{\text {glory }}(\theta)$. Third, the distance of the glory maxima is a function of effective radius and depends only little on the width of the size distribution. Fourth, the details of the glory reflectivity, in particular the appearance of the side maxima is directly related to the width of the size distribution. Utilizing these four features, a remote sensing scheme for three parameters, optical thickness, effective radius, and width of the size distribution from one single wavelength is developed.

For this purpose, tables of the glory reflectivity $R_{\text {glory }}(\theta)$ and the average reflectivity $\langle R(\theta)>$ were calculated for a wide range of effective radii $(4-15 \mu \mathrm{m})$, widths $(0.1-$ $5 \mu \mathrm{m})$, and optical thicknesses (0-50). As explained above, the glory reflectivity depends only on effective radius and width. The tabulated functions are therefore denoted $R_{\text {glory }}\left[r_{\text {eff }}, \sigma\right](\theta)$. An optical thickness of 15 was chosen for the calculation of the glory reflectivity but this particular choice has no influence on the retrieved results. For the retrieval we chose a least-square fitting technique, where a function

$y\left[r_{\text {eff }}, \sigma\right](\theta)=a \cdot \theta+b+c \cdot R_{\text {glory }}\left[r_{\text {eff }}, \sigma\right](\theta)$

is fitted to the data. As the amplitude of the glory reflectivity should only depend on $r_{\text {eff }}$ and $\sigma, c$ would ideally equal 1 . However, due to calibration uncertainties, due to small deviations of the viewing direction from the exact $180^{\circ}$ backscatter direction, and due to differences between the actual size distribution from the assumed gamma function, $c$ may deviate from 1 and hence it is a necessary fit parameter. $c$ is a measure of the quality of the fit and the data.

The first two terms are the multiple scattering background. The second term is basically the average reflectivity while the first term allows some degree of inhomogeneity in the optical thickness, in form of a linear variation of the multiplescattered radiance within the glory region. This is just an arbitrary assumption and could be replaced by a more complex function - however, one has to make sure that the assumed function does not already fit part of the glory structure which is avoided by the simple linear variation. The parameters $a, b$, and $c$ as well as the optimum curve $R_{\text {glory }}\left[r_{\text {eff }}, \sigma\right]$ and hence the effective radius and width of the size distribution are determined by minimizing the cost function

$S\left[r_{\mathrm{eff}}, \sigma\right]=\sum_{i=1}^{N}\left(R\left(\theta_{i}\right)-y\left[r_{\mathrm{eff}}, \sigma\right]\left(\theta_{i}\right)\right)^{2}$,

where the summation is done over all CASI pixels within $\pm 5^{\circ}$ of the $180^{\circ}$ backscatter direction (between $-15^{\circ}$ and $-5^{\circ}$ in the studied case). An example of such a fit is shown in Fig. 5. Scan line 612 was extracted from the CASI observation (see dotted line in Fig. 2) because the cloud seems to be reasonably homogeneous in this region. The thick line

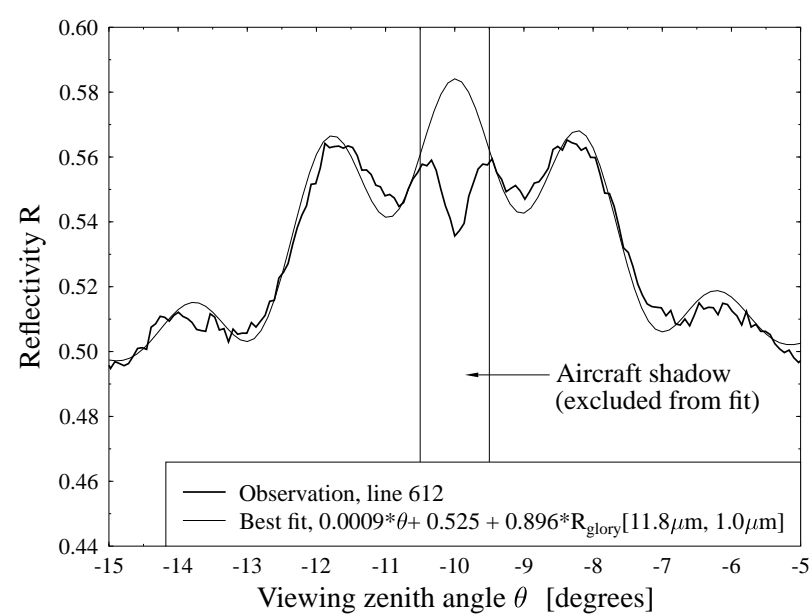

Fig. 5. Example of a fit for scan line 612; the thick line is the observed reflectivity and the thin line is the best fit. The width of the aircraft shadow is about 8 pixel or $20 \mathrm{~m}$ which is close to the actual wing-span of the Do-228 of $17 \mathrm{~m}$.

is the observation, with the aircraft shadow clearly visible in the center. The marked region has been excluded from the fit. The thin line is the best fit.

The glory itself and the first side maximum is almost perfectly fitted by the simulated glory reflectivity for effective radius $11.8 \mu \mathrm{m}$ and width $1 \mu \mathrm{m}$. The scaling factor is reasonably close to $1(0.896)$. The multiple scattering background is described by the first two terms; the linear variation is negligible in this case. The background $a \cdot \theta+b$ is evaluated at the location of the glory to determine the optical thickness using the tabulated values. This means essentially to apply the curve for the determined effective radius and width from the bottom panel of Fig. 3: In our example, using the curve for $r_{\text {eff }}=11.8 \mu \mathrm{m}$, an optical thickness of 13.2 is determined.

To automate the algorithm, another step is neccessary. As Fig. 2 shows, the location of the glory varies slightly in the image which is due to the changing aircraft attitude with respect to the nadir direction and the solar principle plane. The available data for pitch, roll, and yaw of the aircraft were not accurate enough to correct the image with the desired accuracy of one CASI pixel $\left(0.07^{\circ}\right)$. Therefore, a simple edge detection technique was applied to the image, to automatically identify the aircraft shadow and to align the image correspondingly, see Appendix.

The top panel of Fig. 6 shows the stratified CASI observation, after application of the described algorithm. The aircraft motion is completely corrected, and the glory is transformed to a straight line. The lower two plots show the results of our retrieval for the optical thickness and effective radius. The effective radius shows surprisingly little variation, except for regions where large inhomogeneities occur around the $180^{\circ}$ backscatter direction. This demonstrates the robustness of the retrieval. The effective radius is rather smooth in 

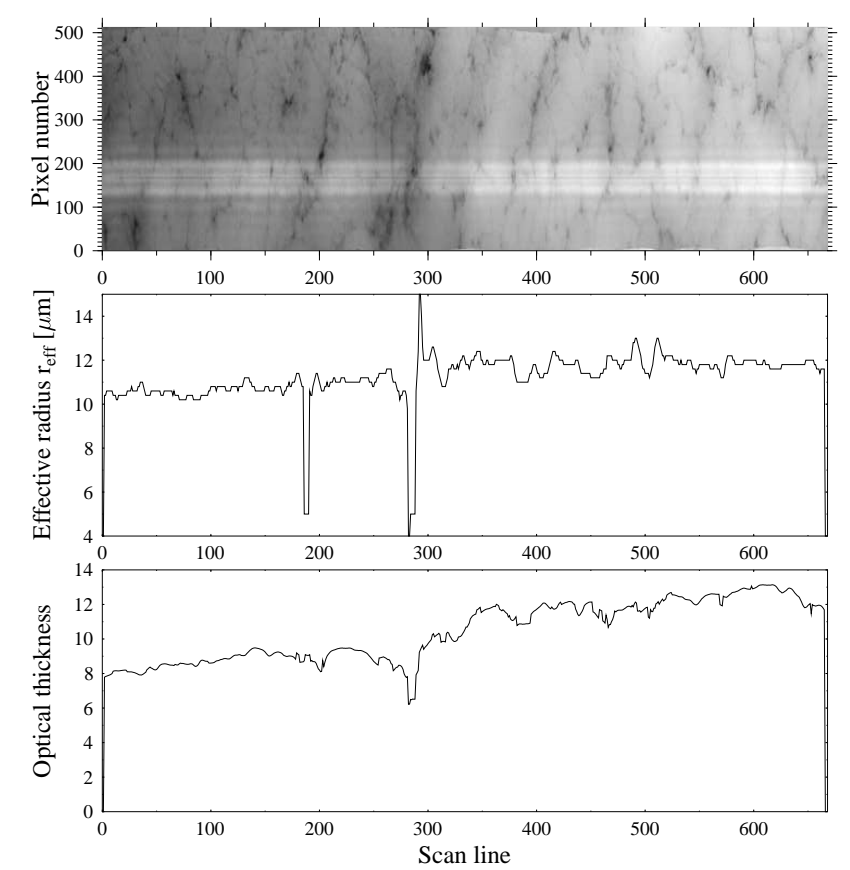

Fig. 6. (Top) Stratified CASI observation; (middle) retrieved effective radius $(\mu \mathrm{m})$; (bottom) retrieved optical thickness.

particular in the left part of the image where the cloud inhomogeneity is small. The effective radius is between 10 and $12 \mu \mathrm{m}$ which is reasonable for this type of boundary layer cloud. The optical thickness is also typical for marine stratocumulus.

The determination of the width of the size distribution from individual scan lines, however, turns out to be not very robust. The reason is immediately obvious from Fig. 4: The information about the width of the size distribution is hidden in small details, e.g. the appearance of the side maxima which in this example vanishes for widths larger than $1.5 \mu \mathrm{m}$. These small details, however, are easily shadowed by the natural variability of the signal, and it is clear that the width of the size distribution cannot be derived from a single scan line. Therefore, the following averaging procedure was chosen: First, the effective radius was determined as in Fig. 6, assuming a constant width of $1 \mu \mathrm{m}$ (the assumption of a constant width has only little impact on the derived effective radius); second, scan lines with identical effective radii were averaged (in bins of width $0.2 \mu \mathrm{m}$ ). This sorting by effective radius reduces the influence of cloud inhomogeneity considerably. Figure 7 shows an exemplary outcome of this procedure. The top panel presents the averaged reflectivity for effective radius $11 \mu \mathrm{m}$ (thick line) which is an average over 50 individual scan lines. In contrast to a single scan line (Fig. 5), the noise is reduced considerably and two secondary maxima at each side of the three main maxima are clearly distinguishable. In comparison to the experimental data (thick line), four fit curves (according to Eq. 8)
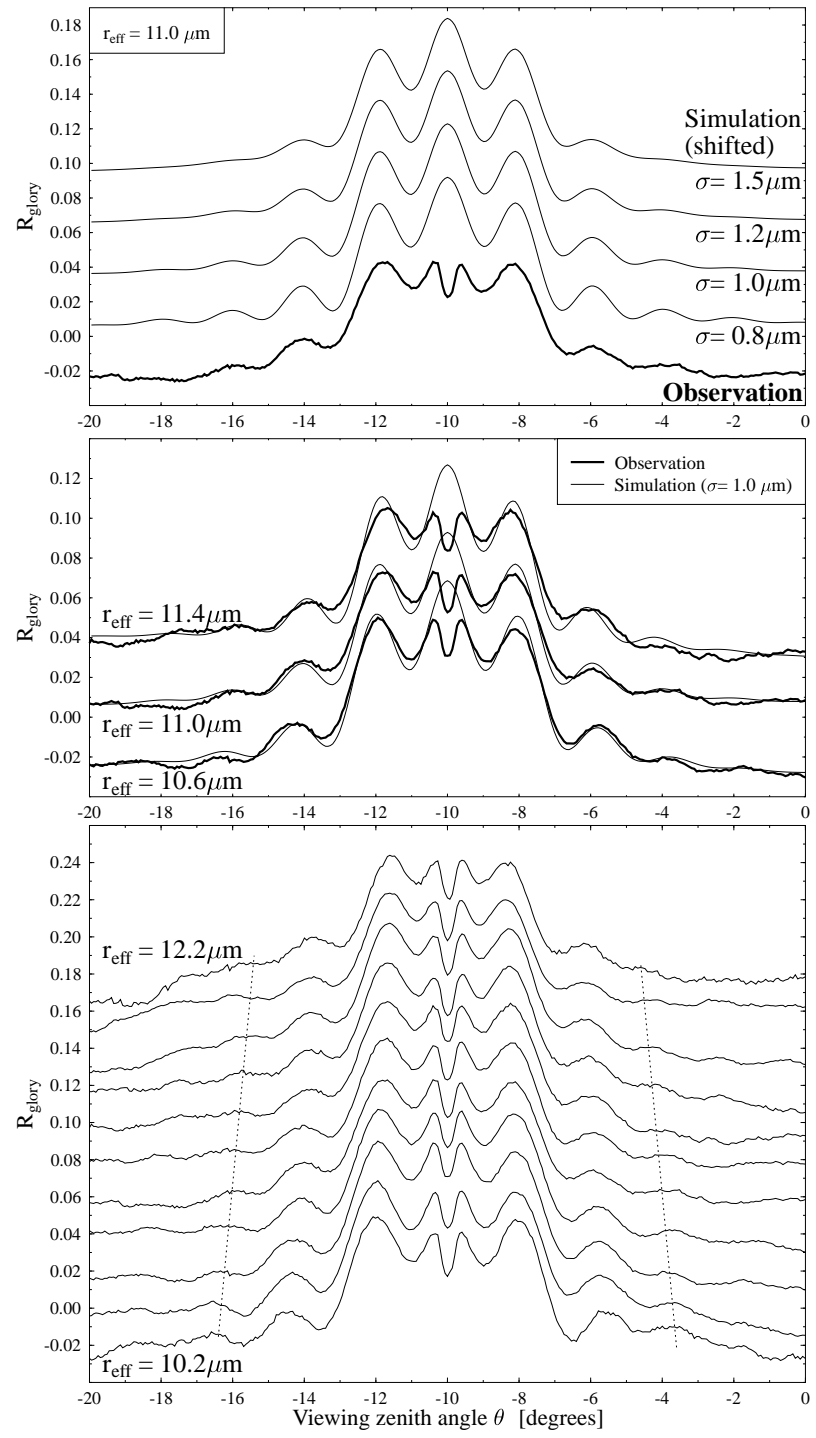

Fig. 7. (Top) Glory reflectivity, averaged over all scan lines with effective radius $11 \mu \mathrm{m}$ (thick line) and simulated glory reflectivities for four different widths of the size distribution; the curves are shifted to allow better distinguishing; (middle) same for three effective radii 10.6, 11.0, and $11.4 \mu \mathrm{m}$, in comparison with the model fit for width $1 \mu \mathrm{m}$; (bottom) averages for all effective radii where more than 10 scan lines were available; the dashed curves indicate the location of the second side-maximum according to the simulation of the glory.

for different widths $0.8,1.0,1.2$, and $1.5 \mu \mathrm{m}$ (thin lines) are presented. Visual comparison of the side maxima suggests a width of $1.0 \mu \mathrm{m}$. For $1.2 \mu \mathrm{m}$ the second side maximum, which is clearly visible in the observation, is hardly distinguishable in the simulation and for $1.5 \mu \mathrm{m}$ it vanished completely. The middle panel shows the results for effective radii 10.6, 11.0, and $11.4 \mu \mathrm{m}$, in direct comparison with the best fit model curves. Finally, the bottom plot presents averages 
for 11 effective radii between 10.2 and $12.2 \mu \mathrm{m}$ (all curves are shown for which more than 10 scan lines were available to calculate the average). For each individual curve, the second side-maxima are clearly distinguishable. The dotted line mark the location of the second side maximum according to the simulation. In some cases, even the third side-maxima are visible. From all this evidence it can be concluded that the width of the size distribution is about $1.0 \mu \mathrm{m}$ for this observation. As the figure illustrates, for widths larger than about $1.2 \mu \mathrm{m}$ the second side-maximum vanishes, while for widths smaller than about $0.8 \mu \mathrm{m}$ the second and third side-maxima are more pronounced than in the observation.

\section{Conclusions}

Based on spatial high resolution observations, a retrieval scheme is presented which allows the simultaneous determination of optical thickness, effective radius, and the width of the droplet size distribution from observations at one single wavelength. The retrieval relies on the observation of the glory in the $180^{\circ}$ backscatter direction at $753 \mathrm{~nm}$. It has been shown by combined Mie calculations and radiative transfer simulations, that the effect of an increasing optical thickness increases the average reflectivity but does not affect the characteristic appearance of the glory. The glory itself depends on the effective radius and the width of the droplet size distribution. While the distance of the maxima is basically a function of the effective radius, the amplitude of the smaller side maxima is determined by the width of the droplet size distribution. In particular, for an effective radius of about $10 \mu \mathrm{m}$, the secondary maxima are only distinguishable if the width of the size distribution is about $1 \mu \mathrm{m}$ or smaller. As this is the case in the example studied here and also in many other observations during this campaign, we conclude that the width of the size distribution was about $1 \mu \mathrm{m}$ which is considerably smaller than what is often assumed for radiation calculations, see Fig. 1. Simultaneous microphysical measurements and theoretical considerations during the ACE-2 CLOUDYCOLUMN campaign confirm the existence of narrow droplet distributions. In particular, Fig. 2 from Schüller et al. (2003) shows a measured droplet spectrum which is very close to the $1 \mu \mathrm{m}$ curve in Fig. 1 . The optical thickness retrieved with our method is at the upper end of the histogram presented in Fig. 2 of Schüller et al. (2003) for the same day, 26 June 1997. The effective radius shows encouraging agreement with the in-situ observations presented in Fig. 1 of Brenguier et al. (2000b), also for 26 June 1997, considering that remote sensing observations of the effective radius are always representative of the upper part of the cloud.

Special features like the rainbow or the glory have been previously exploited for the study of cloud properties. Spinhirne and Nakajima (1994) suggested to use aircraft observations of the glory for remote sensing of droplet sizes. In particular they recommended to use near-infrared observations where the multiple-scattering background is suppressed due to absorption in water droplets. Breon and Goloub (1998) followed a similar approach for the retrieval of effective radius from POLDER observations. They used three spectral channels in the visible and utilized POLDER's ability to measure polarized radiances. With this method they were able to retrieve the effective radius in a field of view between $150^{\circ}$ and $170^{\circ}$. They exploited the sensitivity of the polarized phase function to effective radius and the width of the droplet size distribution, similar to our study. For practical application it has to be kept in mind that the method requires reasonably homogeneous cloud conditions over the required angular range. A variability of optical thickness or effective radius blurs the specific features of the glory, see also (Rosenfeld and Feingold, 2003). In the case of the CASI observation where the aircraft flight altitude was about $2 \mathrm{~km}$ above the cloud, the $10^{\circ}$ which were used for the evaluation translate to only $350 \mathrm{~m}$ over which homogeneity is required. In the case of the low-Earth-orbiting Polder, however, the angular range used in the analysis corresponded to about $150 \mathrm{~km}$ (Breon and Goloub, 1998). It is immediately obvious that the homogeneity criterion is much easier fulfilled for a low-flying aircraft. In fact, the flight altitude of $2 \mathrm{~km}$ above the cloud of the Do-228 in this paper is ideal for this application: a lower flight altitude is desirable to minimize cloud inhomogeneity within the required angular range. On the other hand, a lower flight altitude would cause the aircraft shadow to cover a larger angular range which would prevent a quantitative analysis (see Fig. 5).

The retrieval of optical thickness and effective radius proved to be robust while the width of the size distribution strongly depends on features of small intensity, in particular on the side maxima of the glory. Therefore, averaging scan lines with similar effective radii is essential for the determination of the width of the distribution and improves the quality significantly. However, this averaging procedure implies some loss of information. While effective radius and optical thickness are determined at a very high resolution, the natural variability of clouds prohibits the determination of the width of the size distribution at the same spatial scale. This prevents studies e.g. of the variability of the size distribution within a single cloud cell but still provides very useful information, in particular, the width of the size distribution averaged over the field of view of typical satellite instruments. For future cloud physics campaigns, such measurements should be considered a valuable addition, if the sun is high enough to permit a high-resolution observation of the glory. In case studies, this approach can build a powerful link between microphysical and radiation measurements. For such studies, the scan direction must exactly cross through the $180^{\circ}$ backscatter direction. A small mis-alignment would cause large problems in the evaluation of the data. The quality of the alignment can be easily determined from the visibility of the aircraft shadow in the data. 
A neccessary assumption of the retrieval is the shape of the size distribution. Although the glory carries information about the width of the size distribution, it can certainly not be used to retrieve its exact shape, due to the natural variability of clouds within the glory region which distort the signal. Hence, an a-priori assumption about the shape is required. The retrieved width has therefore to be interpreted as an effective width for an assumed gamma-shape. If the actual distribution differs strongly from the gamma distribution (e.g. a bimodal distribution where both peaks contribute significantly to the scattering phase function), the retrieved width would be meaningless.

Although of little practical relevance, it is interesting to note that, since the glory is a single scattering phenomenon, it is not affected by three-dimensional cloud inhomogeneity effects. Generally, reflected radiance is clearly affected by cloud inhomogeneity and net horizontal photon transport which causes radiative smoothing (Marshak et al., 1995). Hence, the derived optical thickness is artificially smoothed over a scale determined by the physical cloud height. The plane-parallel assumption, made by the retrieval, may also cause an underestimation of the optical thickness (Davis et al., 1997) which affects any cloud retrieval. This is also true for the optical thickness derived by our method. Properties derived from single-scattered glory reflectivity (effective radius and width of the size distribution), on the other hand, are not affected by smoothing and are hence representative for the area used in the retrieval; in our case, $350 \mathrm{~m} \times 75 \mathrm{~m}$.

From the occurrence of the side maxima, we concluded that the width of the droplet size distribution was about $1 \mu \mathrm{m}$ or less for the marine stratocumulus during the ACE-2 CLOUDYCOLUMN experiment. Such a distribution looks conceptually different to what is generally used in radiation studies, see Fig. 1. The top panel shows the commonly employed C1 size distribution by Deirmendjian (1969) in comparison to a size distribution which is more representative of the conditions observed here, in particular, the curve for effective radius $10 \mu \mathrm{m}$ and width $1 \mu \mathrm{m}$. Simultaneous microphysical measurements and theoretical considerations during the ACE-2 CLOUDYCOLUMN campaign confirm the existence of narrow droplet distributions (Schüller et al., 2003).

\section{Appendix: Image alignment}

To correct the image for aircraft misalignment we developed a technique which automatically identifies the position of the aircraft shadow. As Fig. 5 shows, the aircraft shadow is the region with the strongest gradient in the image. The Sobel edge detection algorithm (see e.g. Cherri and Karim, 1989) was chosen to identify the strongest horizontal gradients in the image. The data is convolved with a $3 \times 3$ matrix

$$
\left[\begin{array}{ll}
-1 & 0+1 \\
-2 & 0+2 \\
-1 & 0+1
\end{array}\right]
$$

which is similar to a derivative. The result is large when the gradient perpendicular to the flight track is large. Numbers smaller than zero indicate negative gradients, numbers larger than 0 positive ones. For the presented example the minima and maxima of the convolved image marked the left and right side of the aircraft shadow in more than $75 \%$ of all scan lines. Cloud edges which are present in several parts of the image, may cause problems with this method. Therefore, we introduced the following two extra requirements: (1) maximum and minimum gradient are required to lie within certain bounds; a histogram of distances between minimum and maximum is created, and all data points are excluded where this distance deviates by more than 2 pixels from the histogram maximum; this way, the width of the aircraft shadow is determined dynamically for the image; (2) for all the scan lines that fulfil the first criterion, the $180^{\circ}$ backscatter direction is assumed to lie midway between the minimum and maximum gradients; from this set of data points those are excluded which deviate by more than a certain threshold from the linear interpolation between the adjacent scan lines; for the example in Fig. 2 this threshold was set to 1 pixel. Finally, the gaps are filled by linear interpolation between the thus determined points. Although these criterions seem rather strict, 506 out of 668 scan lines remained to determine the location of the $180^{\circ}$ backscatter direction. The result is shown in Fig. 6 which clearly illustrates the potential of the method.

Acknowledgements. M. Schröder was supported by the German BMBF project 4D-Clouds under contract number 07ATF24.

Edited by: A. Lambert

\section{References}

Anderson, G., Clough, S., Kneizys, F., Chetwynd, J., and Shettle, E.: AFGL Atmospheric Constituent Profiles (0-120 km), Tech. Rep. AFGL-TR-86-0110, AFGL (OPI), Hanscom AFB, MA 01736, 1986.

Anger, C., Mah, S., and Babey, S.: Technological enhancements to the Compact Airborne Spectrographic Imager (casi), in Proceedings of the Second International Airborne Remote Sensing Conference and Exhibition, 12-15 September, Strasbourg, France, pp. 205-214, 1994.

Babey, S. and Anger, C.: A compact airborne spectrographic imager (casi), in Proceedings of IGARSS, IEEE, Vancouver, vol. 2, pp. 1028-1031, 1989.

Brenguier, J., Chuang, P., Fouquart, Y., Johnson, D., Parol, F., Pawlowska, H., Pelon, J., Schüller, L., Schröder, F., and Snider, J.: An overview of the ACE-2 CLOUDYCOLUMN closure experiment, Tellus B, 52, 815-827, 2000.

Brenguier, J.-L., Pawlowska, H., Schüller, L., Preusker, R., Fischer, J., and Fouquart, Y.: Radiative properties of boundary layer clouds: Droplet effective radius versus number concentration, J. Atm. Sci, 57, 803-821, 2000. 
Breon, F.-M. and Goloub, P.: Cloud droplet effective radius from spaceborne polarization measurements, Geophys. Res. Lett., 25, 1879-1882, 1998.

Cherri, A. and Karim, M.: Optical symbolic substitution: edge detection using Prewitt, Sobel, and Roberts operators, Applied Optics, 28, 4644-4648, 1989.

Cox, C. and Munk, W.: Measurement of the roughness of the sea surface from photographs of the sun's glitter, Journal of the Optical Society of America, 44, 838-850, 1954.

Davis, A., Marshak, A., Cahalan, R., and Wiscombe, W.: The Landsat scale break in stratocumulus as a three-dimensional radiative transfer effect: Implications for cloud remote sensing, J. Atmos. Sci., 54, 241-260, 1997.

Deirmendjian, D.: Electromagnetic Scattering on Spherical Polydispersions, American Elsevier Publishing Company, Inc., New York, 1969.

Hansen, J. E. and Travis, L. D.: Light scattering in planetary atmospheres, Space Sci. Rev., 16, 527-610, 1974.

Houghton, J., Ding, Y., Griggs, D., Noguer, M., van der Linden, P., Dai, X., Maskell, K., and Johnson, C.: Climate change 2001: The scientific basis, Intergovernmental Panel on Climate Change (IPCC), IPCC Secretariat, c/o World Meteorological Organization, Geneva, Switzerland, 2001.

Kylling, A. and Mayer, B.: Libradtran, a package for radiative transfer calculations in the ultraviolet, visible, and infrared, http://www.libradtran.org, 1993-2004.
Laven, P.: Simulation of rainbows, coronas, and glories by use of Mie theory, Applied Optics, 42, 436-444, 2003.

Marshak, A., Davis, A., Wiscombe, W., and Cahalan, R.: Radiative smoothing in fractal clouds, J. Geophys. Res., 100, 26247 $26261,1995$.

Nakajima, T. and Tanaka, M.: Effect of wind-generated waves on the transfer of solar radiation in the atmosphere-ocean system, J. Quant. Spectrosc. Radiat. Transfer, 29, 521-537, 1983.

Rosenfeld, D. and Feingold, G.: Explanations of discrepancies among satellite observations of the aerosol indirect effects, Geophys. Res. Lett., 30, doi:10.1029/2003GL017684, 2003.

Schüller, L., Brenguier, J.-L., and Pawlowska, H.: Retrieval of microphysical, geometrical, and radiative properties of marine stratocumulus from remote sensing, J. Geophys. Res., 108, doi:10.1029/2002JD002680, 2003.

Spinhirne, J. and Nakajima, T.: Glory of clouds in the near infrared, Applied Optics, 33, 4652-4662, 1994.

Stamnes, K., Tsay, S., Wiscombe, W., and Jayaweera, K.: A numerically stable algorithm for discrete-ordinate-method radiative transfer in multiple scattering and emitting layered media, Applied Optics, 27, 2502-2509, 1988.

Wiscombe, W.: Improved Mie scattering algorithms, Applied Optics, 19, 1505-1509, 1980. 